\title{
Computational identification and functional annotation of miRNAs in medicinal plant Helianthus petiolaris
}

\author{
Sarika Sahu • Dev Bukhsh Singh · Kusum Kumari Yadav • \\ D. V. Rai $\cdot$ Rekha Dixit
}

Received: 16 February 2013/Revised: 23 September 2013/Accepted: 16 October 2013/Published online: 16 November 2013

(C) Springer-Verlag Wien 2013

\begin{abstract}
MicroRNAs (miRNAs) are short ( 22 nucleotide), non-coding RNAs that regulate messenger RNA (mRNA) or protein levels either by promoting mRNA degradation or by attenuating protein translation. Computation identification of miRNAs and their targets in medicinal plant Helianthus petiolaris (H. petiolaris) was carried out. Computational approach for mining of miRNA from EST databases is faster and reliable. Here, the total 27,484 EST sequences of $H$. petiolaris were assembled and clustered into 4,182 contigs (15\% of EST sequences). These contigs were searched against known miRNA sequences of different plants. An overall 117 unique sequences (precursor miRNAs) were mined from UniGenes. Functional annotation of unique sequences was carried out to eliminate the coding sequences and secondary structure of resulting precursor miRNAs (pre-miRNAs) was determined. The analysis led to identification of 11 miRNAs sequences (10\% of pre-miRNAs). The hpe-mir395 was found to be the most stable miRNA on the basis of free energy of secondary structure. Targets for novel miRNAs were determined by searching homology between identified miRNA and UniGenes. Eleven miRNA targets
\end{abstract}

Electronic supplementary material The online version of this article (doi:10.1007/s13721-013-0044-8) contains supplementary material, which is available to authorized users.

S. Sahu $(\bowtie) \cdot$ K. K. Yadav · D. V. Rai · R. Dixit

Faculty of Biological Engineering, Shobhit University,

Meerut 250110, Uttar Pradesh, India

e-mail: sahusarikaiiita@gmail.com

D. B. Singh

Department of Biotechnology, Institute of Biosciences and

Biotechnology, Chhatrapati Shahu Ji Maharaj University,

Kanpur 208024, Uttar Pradesh, India

e-mail: answer.dev@gmail.com were identified of which $67 \%$ are responsible for biological function. The predicted miRNAs were validated by phylogenetic study and belong to four large miRNA families (mir-393, mir-156, mir-1863, mir-169).

Keywords Prediction - miRNA - EST - Target identification $\cdot$ Computational $\cdot$ Annotation

\section{Introduction}

An extensive population of small RNA molecules that have the capacity to regulate gene expression at the post-transcriptional level has been identified recently in plants (Yao et al. 2007; Varkonyi-Gasic et al. 2010; Sahu and Dixit 2011; Yeo and Chong 2011). miRNAs play key regulatory roles in many cellular processes such as differentiation, replication and regeneration of cell. Because of these regulatory roles, miRNA expression has been implicated in several diseases. Initially this work has been carried out in Caenorhabditis elegans in the early 1990s (Lee et al. 1993), but here since been reported in a different organisms ranging from single cell (algae) to complex humans, suggesting that miRNAs are ancient and critical cellular regulatory element in biological function (Bartel and Chen 2004; Zhao et al. 2007).

It is estimated that up to $40 \%$ of miRNAs are produced from introns of protein-coding genes and thus are transcribed with the help of host genes (Kong and Han 2005). Mitrons are a form of microRNAs generated from spliced introns. However, majority of miRNAs are derived from independent transcriptional units instead of spliced introns. These primary miRNA (Pri-miRNAs) transcripts are several hundred nucleotides long and are modified similar to protein-coding transcripts by the addition of a $5^{\prime}$ cap and a 
$3^{\prime}$ poly-A tail (Etheridge et al. 2011). Pri-miRNAs are first processed in the nucleus and later in the cytosol by RNase III enzyme Drosha and Dicer, respectively (Lee et al. 2003). RNA-folding programmes are used to analyze the structure of putative transcripts from which the miRNAs could be identified (Zuker 2003).

In silico identification of miRNAs has gained momentum because miRNAs are evolutionarily conserved. There are four approaches for identifying miRNAs: (1) genetic screening, (2) direct cloning after isolation of small RNAs, (3) computational strategy and (4) expressed sequence tags (ESTs) analysis (Sahu et al. 2011). miRNAs have been identified through homology searching (Lu and Yang 2010). miRNA has been predicted in HIV viruses using artificial neural network approach (Gupta et al. 2012a, b). A decision-tree model for miRNA prediction has been developed by supervised machine learning. It is based on most critical features for training: the duplex energy (miRNA:miRNA) and the number of mismatches in the duplex (Philip et al. 2012). Nowadays, ESTs have been utilized for identification of plant miRNAs and their targets (Liang et al. 2010; Jha and Shankar 2011; Shallu and Sahu 2011). It is reported that integrating ESTs (flanking structural RNA element) improves the quality of cleaved miRNA predictions and suggests that this approach can be used for prediction of other non-coding RNAs undergoing cleavage during maturation (Krzyzanowski et al. 2011).

Wild Helianthus species have been a reliable source of genes for resistance to economically important pathogens. These species are resistant to rust, downy mildew, Verticillium wilt, Alternaria leaf spot, Powdery mildew, Phomopsis stem canker, Sclerotinia wilt/rot, and parasitic weed broomrape. One of the species is $H$. petiolaris. which is completely resistant to downy mildew (Seiler 2010). H. petiolaris also known as prairie sunflower is a shrub belonging to the family Asteraceae and are tap-rooted annuals. H. petiolaris is a source of dominant genes for single race resistance (Miller and Gulya 2001). Major goal of plant breeders is to incorporate the disease resistance character into cultivated sunflower. The seeds of the plant are edible and can be used in oily meal or butter. The powdered leaves of sunflower are useful in healing of sores and swelling.

Rapid progress in genomics and bioinformatics has transformed the world of biology in recent years. Sequencing of eukaryotic genomes has led to better understanding of gene functions and regulation. Sequencing of plant genomes Arabidopsis, rice and poplar is unraveling new insights for their improvements. H. petiolaris is a plant of great medicinal value which needs to be more explored. However, its genome remains largely unexplored and the only information related to its genome is in the form of 27,484 ESTs in dbEST. Comparative genomics and data mining tools were employed to identify miRNAs as well as their targets. Identification of miRNA using computational approaches is emerging as faster and reliable method as compared to laboratory-based cloning approaches. The scope of computational methods is increasing due to low cost, high accuracy and new mining techniques (Wegener et al. 2013).

\section{Materials and methods}

\subsection{Database used}

Plant miRNAs are highly conserved among distantly related species. miRNAs of known plants Arabidopsis, Oryza, Citrus, Pinus, Triticum, Zea, Brassica and other plant species were downloaded from the miRBase database release 17 (Kozomara and Griffiths-Jones 2011). Known Plant miRNAs were used to search homology between UniGene of $H$. petiolaris for mining of miRNAs. ESTs were used as starting point for the identification of miRNAs in H. petiolaris. Total 27,484 ESTs of H. petiolaris were retrieved from dbEST (Boguski et al. 1993). dbEST is a division of GenBank that contains sequence data of ESTs from a number of organisms (http://www.ncbi.nlm.nih.gov/ $\mathrm{dbEST} /$ ). Availability of the complete genomic sequences of Arabidopsis was the key for predicting the identity of the stem-loop precursor RNAs. The schematic representation of methodology is shown in Fig. 1.

To minimize the redundancy by clustering and assembly, cleaned and trimmed EST contigs were obtained from the EGassembler tools and then processed via Repeatmasker (for masking the repeated sequences) (MasoudiNejad et al. 2006). EGassembler is an online server (http:// egassembler.hgc.jp/), which provides an automated analysis tool for cleaning, vector trimming, repeat and organelle masking, clustering and assembling of ESTs. Local databases of contigs (assembled ESTs and genomic fragments) named HPEST were created using Bioedit sequence alignment editor tool version 7.0.9.0 (Hall 1999).

\subsection{Precursor miRNA identification}

Precursor miRNAs (pre-miRNAs) were identified on the basis of two parameters namely, expectation value ( $E$ value) and word-match size between queries and databases. Homology between miRNA sequences from miRBase and UniGenes from $H$. petiolaris was determined through BLASTN. UniGenes computationally identifies transcripts that appear to arise from same transcription locus (gene or expressed pseudogene). A criterion used for finding homology was an $E$ value cut off 0.001 and wordmatch length nine between query miRNA and Unigene 
Fig. 1 Schematic representation of steps in miRNA prediction and its functional annotation

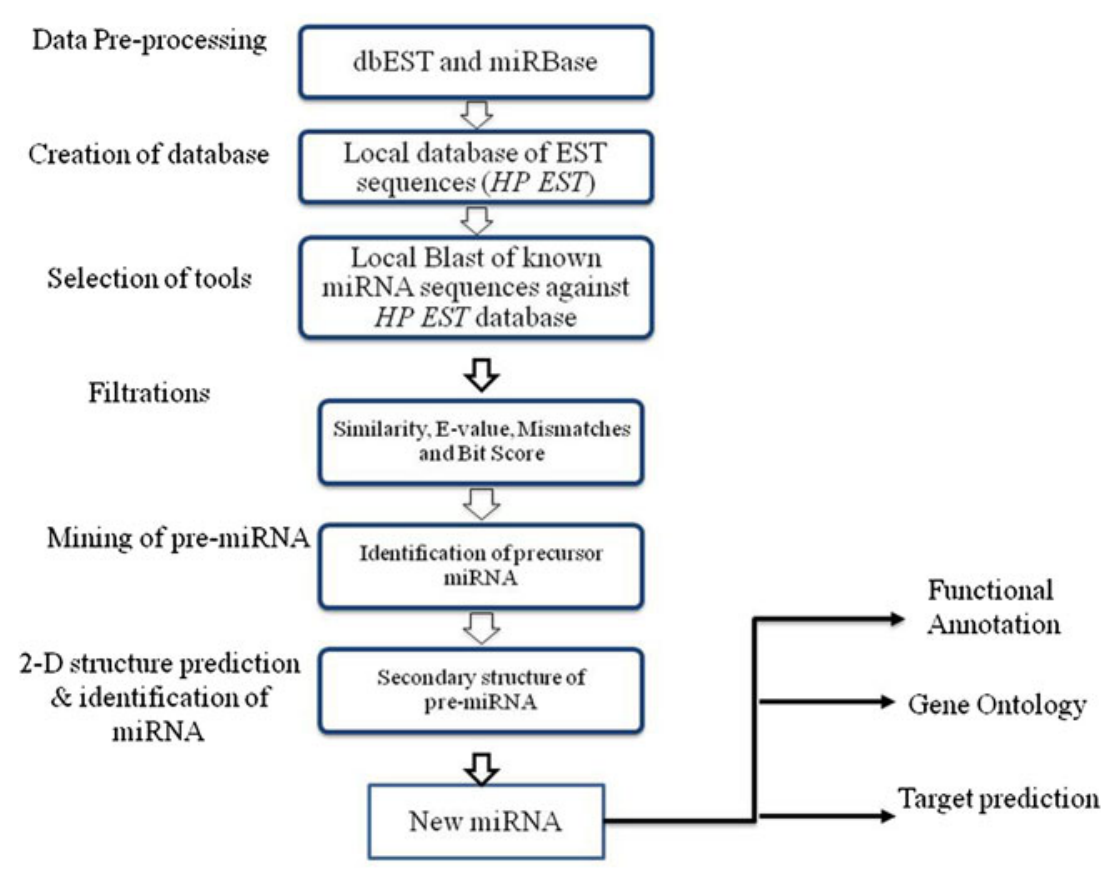

sequences. Basically, the lengths of pre-miRNAs were reported between 70 and 90 nucleotides, which fold into imperfect stem-loop structures (Pfeffer and Voinnet 2006). On the basis of reported length of Pre-miRNA, we fetched 100 sequences upstream and 100 sequences downstream from the matching region. Local programs in perl script facilitated the fetching of Pre-miRNA sequences from the UniGenes (http://www.perl.org/).

Identified precursors from $H$. petiolaris were subjected to folding via mfold server (http://mfold.rna.albany.edu/) and these hairpin structures were manually examined for screening. Criteria used for screening of pre-miRNA were $<8$ unpaired nucleotides and no more than three consecutive unpaired nucleotides of which no more than two asymmetrically bulged in the 16-25 bp stem encompassing the mature miRNA sequence.

\subsection{Extraction of miRNAs from precursor}

Criteria used for miRNA identification from pre-miRNAs are: (a) The pre-miRNA sequence folding into an appropriate stem-loop hairpin secondary structure, (b) a mature miRNA sequence located in one arm of the hairpin structure, (c) predicted mature miRNAs with no more than three nucleotide substitutions as compared to the known miRNAs, (d) miRNAs having less than six mismatches with the opposite miRNA sequence in the other arm, (miRNA*), (e) no loop or break in miRNA* sequences, and (f) predicted secondary structures with higher minimal folding free energy (MFE) and minimal folding free energy index (MFEI), MEFI usually being over 0.85 (Zhang et al. 2006).
The AU content of pre- miRNA within 30-70\% was also considered as parameter for miRNA prediction (Xie et al. 2007).

\subsection{Target identification and functional annotation}

Novel identified $H$. petiolaris miRNAs were used as query sequence against the HPEST database. Alignments between each miRNA and its putative miRNA target were evaluated using following parameters based on complementarity between them: (a) no more than four mismatches were allowed between the mature miRNA and its target site, (b) no more than one mismatch was allowed at nucleotide positions $1-9$, (c) no more than two consecutive mismatches were allowed, (d) no mismatches were allowed at positions ten and eleven (Xie et al. 2010).

Pre-miRNAs were functionally annotated with the help of SwissProt protein database using BLASTX program (http://ncbi.nlm.nih.gov/blast/Blast). Putative function of the UniGenes could provide meaningful information about the biological processes that are regulated by miRNA in $H$. petiolaris. To understand the molecular function and biological role of the predicted miRNA target genes, the miRNA targets have been searched in gene ontology (http://www.geneontology. org). For homology-based functional annotation of UniGenes containing miRNA targets, BLASTX against non-redundant data set, Genbank coding sequence (CDS) translations, protein data bank (PDB), SwissProt, protein information resource (PIR) and protein resource foundation (PRF) was performed. 


\subsection{Validation of predicted miRNAs}

Predicted miRNAs were validated by sequence alignment and phylogenetic study. All novel candidate miRNAs were searched against non-coding RNA database (http://www. ncrna.org) and miRBase database. All these candidate miRNAs were BLAST against EST sequences of $H$. petiolaris. Phylogenetic study was done to compare predicted miRNA with experimentally validated miRNA homologues considering the conservation of miRNA and their precursors. Precursor sequences of the predicted and validated miRNA in the same family were aligned and analyzed by MEGA (version: 5.2.2) software to investigate their evolutionary relationships (Tamura et al. 2011).

\section{Results and discussion}

\subsection{Identified miRNAs in H.petiolaris}

In the present study, publicly available data of $H$. petiolaris 27,484 expressed sequence tagged sequences in dbEST have been assembled and clustered into 4,182 non-

Fig. 2 Secondary structure of H. pteolaris pre-miRNAs a hpemir-395, b hpe-mir-1863 and c hpe-mir-156b. Sequences within red braces in each precursor miRNA may serve as miRNA (color figure online)

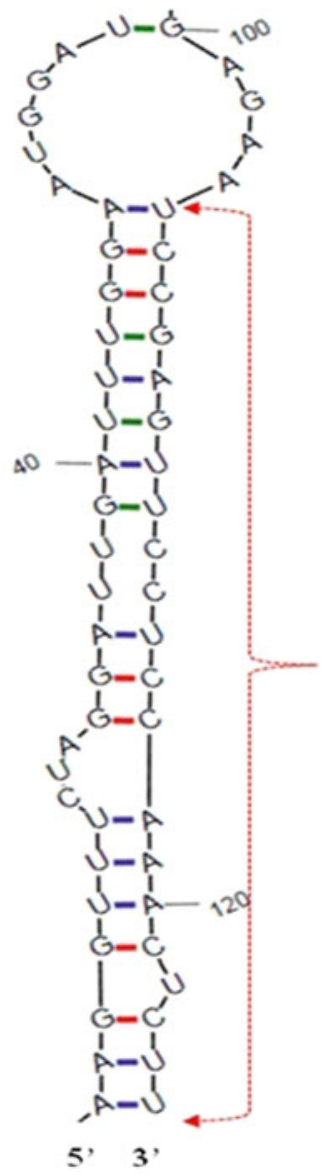

(a) redundant sequences using EGassembler program. This result indicates $85 \%$ data redundancy and EST contigs are only $15 \%$ (i.e., 4,182 of total ESTs) with the help of computational method. Local BLAST of known miRNAs against HPEST database was carried out to determine homology between query miRNA from mirBASE and HPEST database. An $E$ value cut off (0.001) and wordmatch length nine between query miRNA and UniGene sequences was used for screening of potential UniGenes. Finally, eleven (11) Pre-miRNA sequences were fetched out by taking 100 sequences upstream and 100 sequences downstream from the matching region of UniGenes (Supplementary Table 1). Secondary structure analysis of identified pre-miRNA sequences was done to evaluate their stability. The hpe-mir-395, hpe-mir-1863 and hpe-mir$156 \mathrm{~b}$ were found as most stable pre-miRNAs (Fig. 2).

A large number of significant parameters were used for retrieval of miRNA from pre-miRNA sequence. Finally, 11 potential miRNAs were found from each precursor (pre-miRNA) and a unique name was assigned to each identified miRNA. Nomenclatures of these miRNAs were given on the scheme proposed by Ambros (Ambros et al. 2003). Names were assigned to the

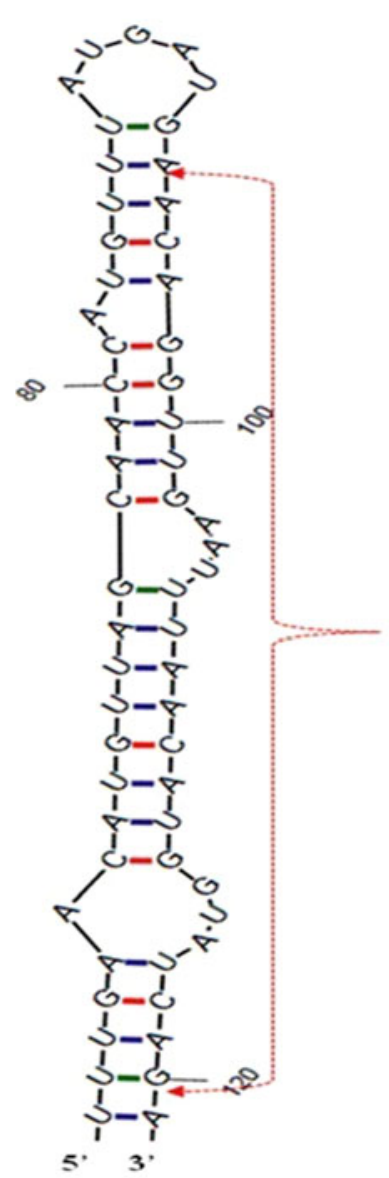

(b)

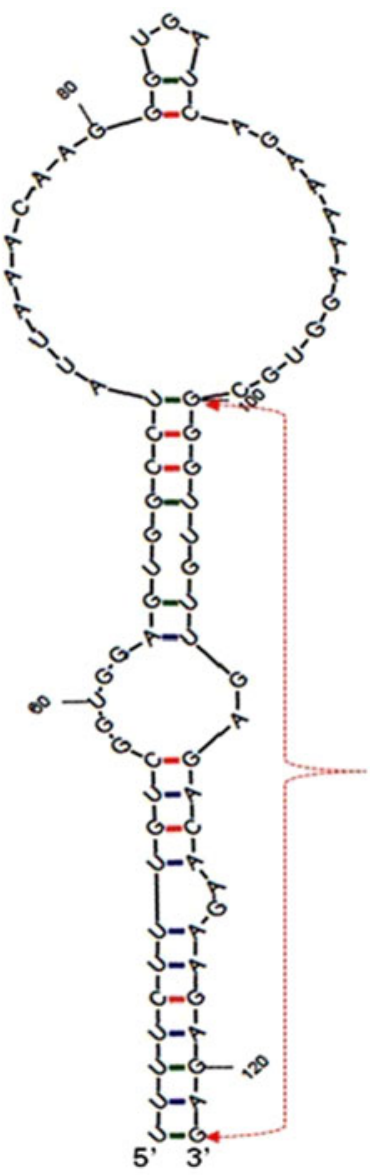

(c) 
Table 1 Description of miRNAs identified in H. petiolaris

\begin{tabular}{|c|c|c|c|c|c|c|c|}
\hline S. no & miRNA & Gene ID & Source & $\mathrm{AU}(\%)$ & miRNA sequence & ORF & Free Energy \\
\hline 1 & hpe-mir-395 & 90477206 & EST & 52.38 & 5'UCCGAGUUCCUCCAAACUCUU3' & Yes & -71.15 \\
\hline 2 & hpe-mir-5272 & 90479661 & EST & 57.14 & 5'CGGAUGAGGAUUAUGUUUGGA3' & No & -68.1 \\
\hline 3 & hpe-mir-156a & 90490255 & EST & 19.05 & 5'GCUCACGGCGGCGGCGGUGGA3' & Yes & -64.94 \\
\hline 4 & hpe-mir-1863 & 90487567 & EST & 71.43 & 5'AACAGGUUGAAUUUAACAUGGUAUCAGA3' & No & -64.69 \\
\hline 5 & hpe-mir- 845 & 90473694 & EST & 57.14 & 5'GGUUACCUCUCUGAUACCAAA3' & Yes & -57.98 \\
\hline 6 & hpe-mir-397 & 90479321 & EST & 42.86 & 5'UCGGCUCGUUAUCGCACCUUG3' & No & -53.09 \\
\hline 7 & hpe-mir-156b & 90488705 & EST & 52.38 & 5'GGGUUGUUGAGACAAGAAGAGAG3' & No & -49.63 \\
\hline 8 & hpe-mir-164 & 90481523 & EST & 57.14 & 5'CUUUUGAAGAGAAGCAGGGUA3' & No & -49.08 \\
\hline 9 & hpe-mir- 1542 & 90482963 & EST & 71.43 & 5'UUUCUGUAUCACUUAUCAAUC3' & No & -48.82 \\
\hline 10 & hpe-mir-5143 & 90486585 & EST & 66.67 & 5'UUCGAUAUAUGUUGGCAAUGA3' & Yes & -45.3 \\
\hline 11 & hpe-mir-169 & 90486484 & EST & 57.14 & 5'UGGAGGAACAAAGAUGACUUG3' & No & -44.04 \\
\hline
\end{tabular}

Fig. 3 Free energy (kcal/mol) of identified miRNAs in $H$. petiolaris

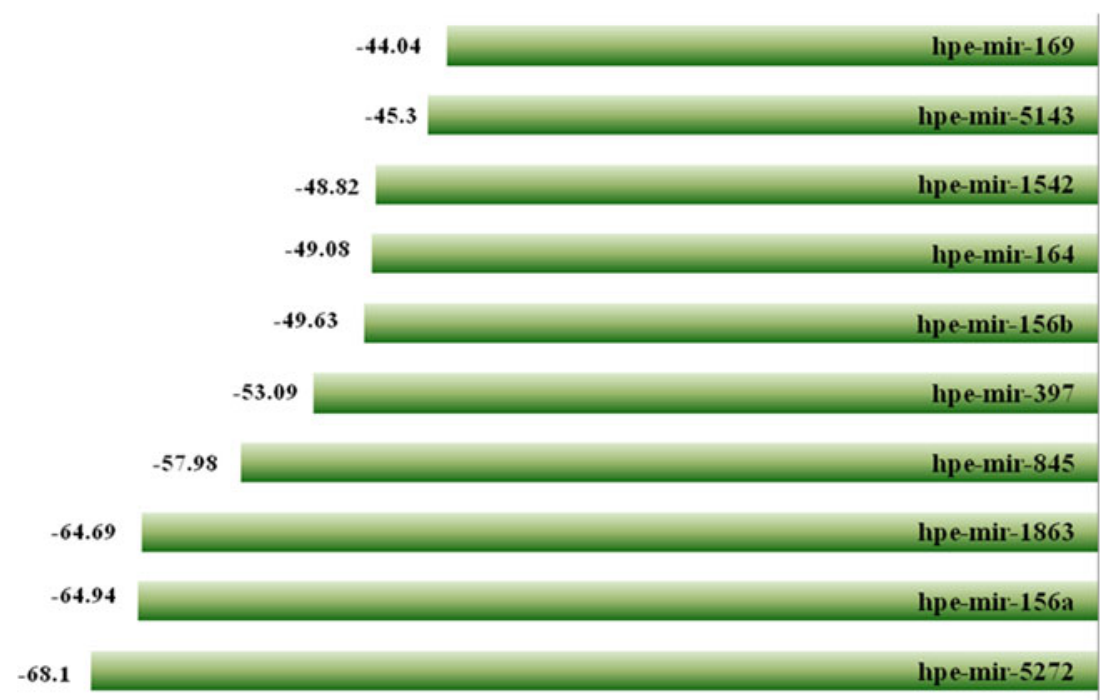

miRNAs in similar pattern to miRBase (Griffiths-Jones et al. 2006). Mature sequences are designated 'miR' and the precursor hairpins are labeled as 'mir' with the prefix "hpe" for $H$. petiolaris. Novel miRNAs of $H$. petiolaris were named as 'hpe-mir-395', 'hpe-mir-5272', 'hpe-mir156a', 'hpe-mir-1863', 'hpe-mir-845', 'hpe-mir-397', 'hpe-mir-156b', 'hpe-mir-164', 'hpe-mir-1542', hpe-mir5143' and 'hpe-mir-169' (Table 1). The free energy of different miRNAs of $H$. petiolaris was determined for analyzing its structural stability (Fig. 3). The hpe-mir395 has lowest energy $(-71.15 \mathrm{kcal} / \mathrm{mol})$, whereas 'hpemir-169' possesses highest energy $(-44.04 \mathrm{kcal} / \mathrm{mol})$ among all miRNAs. All miRNAs have shown favorable free energy (negatively high) between -71.15 and $-44.04(\mathrm{kcal} / \mathrm{mol})$ and could be considered as stable miRNA and may have potential role in biological, cellular and molecular mechanism.

\section{2 miRNA targets and functions}

To understand the biological function of miRNAs in $H$. petiolaris, the putative target sites for the miRNA candidates were identified. Based on the sequence complementarity between the miRNA and their target in plants, identified miRNA was searched against HPEST database using BLASTN tool for finding its potential targets. In this study, we identified $92 \%$ (11) UniGenes as putative targets for 11 conserved miRNAs. Identified target genes in H. petiolaris were found similar or related with the previously validated plant miRNA targets in Arabidopsis. The target UniGenes showed homology with proteins like ATP sulfurylase carnitine transporter. Annotated genes were categorized functionally into three major properties: cellular function, molecular function and biological process in accordance with gene ontology systems. Most of the target 
Table 2 Potential target for miRNA in $H$. petiolaris and their function

\begin{tabular}{|c|c|c|c|}
\hline S. no. & miRNA & Target protein & Function \\
\hline 1 & hpe-mir-395 & ATP sulfurylase-1 & Biological function: sulfur metabolism. \\
\hline 2 & hpe-mir-5272 & Alpha-trehalose phosphate synthase & $\begin{array}{l}\text { Molecular function: controls the over expression of citric } \\
\text { acid. Responsive to acid stress }\end{array}$ \\
\hline 3 & hpe-mir-1863 & $\begin{array}{l}\text { Ethylene-responsive transcription } \\
\text { factor-5 }\end{array}$ & Cellular function: promoting adaptation to drought and salt tolerance \\
\hline 4 & hpe-mir- 845 & Carnitine transporter- 4 & Biological function: response to cold stress \\
\hline 5 & hpe-mir-397 & Laccase-4 & Biological function: carbon stress condition or low-carbon medium \\
\hline 6 & hpe-mir-156b & Ras-related protein RABG1 & Biological function: involves in salt stress \\
\hline 7 & hpe-mir-164 & Uncharacterized oxidoreductase & Biological function \\
\hline 8 & hpe-mir-1542 & NADH-cytochrome b5 reductase- 1 & Molecular function: response in salt stress \\
\hline 9 & hpe-mir-5143 & Polyadenylate-binding protein-2 & Biological function: response in salt stress \\
\hline 10 & hpe-mir-169 & Acid phosphatase-1 & Molecular function: response to phosphate starvation, $\mathrm{ABA}$ and salt stress \\
\hline
\end{tabular}

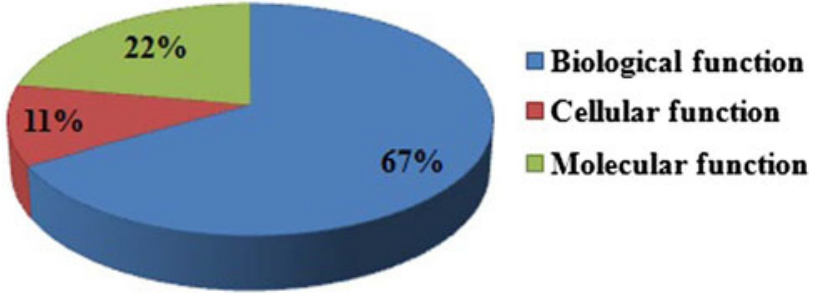

Fig. 4 Functional characterization of miRNAs identified in $H$. petiolaris (color figure online)

miRNA, approximately $67 \%$, are responsible for biological process and $22 \%$ and $11 \%$ participated in cellular and molecular function, respectively (Table 2; Fig. 4).

In functional annotation, majority of prairie sunflower miRNA targets were found to be related with stress response. Identified target ATP sulfurylase plays a very important role in sulfur metabolism of plants. Sulfur nutrition is a component contributing towards defense mechanism of plant (Rausch and Wachter 2005). Another target, alpha-trehalose phosphate synthase is contributed in citric acid stress response in yeast and over expression of this enzyme in common bean led to the increase in drought tolerance (Lawrence et al. 2004; Suárez et al. 2008). In yeast, gene expression profiling in response to citric acid stress resulted in up regulation of alpha-trehalose phosphate synthase indicating its role as a stress response gene in yeast.

\subsection{Phylogeny of predicted miRNAs}

BLASTS of candidate miRNAs against EST sequences have shown positive hits for all predicted miRNAs. Some miRNAs were present in open reading frame (ORF) region which indicates that these miRNAs were processed from these
ESTs. The newly identified eleven $H$. petiolaris precursor miRNAs belong to four large miRNA families (mir-393, mir-156, mir-1863, mir-169). Two candidates of $H$. petiolaris precursor miRNA were found in same family (hpe-mir$156 \mathrm{a}$ and hpe-mir-156b). The hpe-mir-156 is closely related with the osa-MIR156h and osa-MIR156f of Oryza sativa (Fig. 5a). Phylogenetic tree for mir-169 family (Fig. 5b) has shown that aly-MIR169n from Arabidopsis lyrata has close evolutionary relationship with hpe-mir-169. The hpe-mir395 has shown similarity and evolutionary relationship with miRNAs reported in many other plant species (Fig. 5c). Similarly, predicted hpe-mir-1863 is in very close evolutionary relationship with osa-MIR 1863a (Oryza sativa) (Fig. 5d) Phylogenetic tree of four families clearly indicates evolutionary relationship of predicted precursor miRNAs with those of other. Comparison of precursor miRNA homologues has shown that all members have high sequence similarity and also reveal evolutionary conservation of precursor miRNAs. The accuracy of predicted precursor miRNAs was validated by their conserved nature and evolutionary relationship with experimentally reported precursor miRNAs in other plant species.

A major goal for agriculture is to develop stress tolerance and yield in crops. As our objective plant has resistance gene against some pathogens, the incorporation of disease resistance into cultivated sunflower could be a major goal of plant breeders. In this study, 11 potential miRNAs and their targets were identified in prairie sunflower. Most of the miRNA targets are related with stress responses such as enzyme Alpha-trehalose phosphate synthase that controls the over expression of citric acid, ATP Sulfurylase participate to enhance the self defense mechanism. The findings suggest that identified miRNAs of $H$. petiolaris are involved in regulation of genes related 

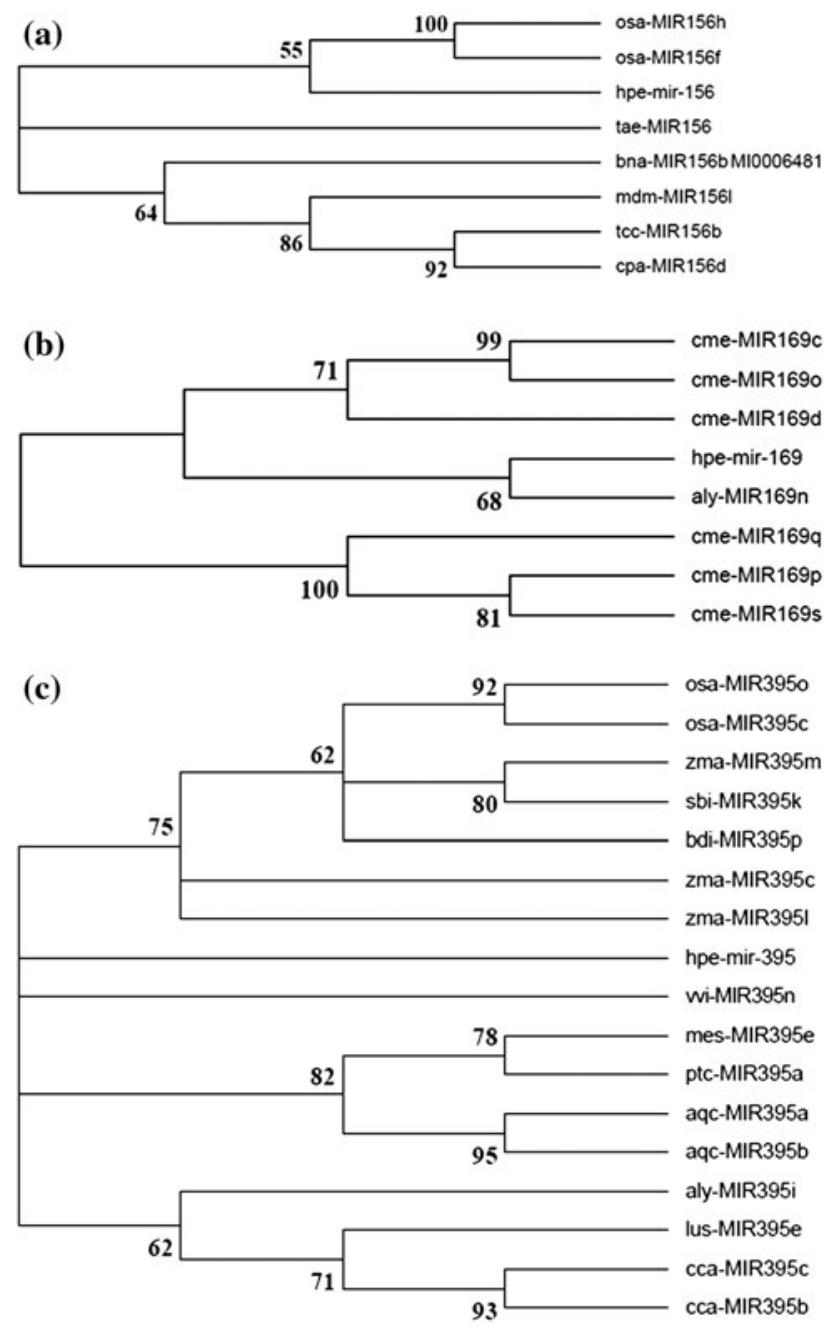

(d)

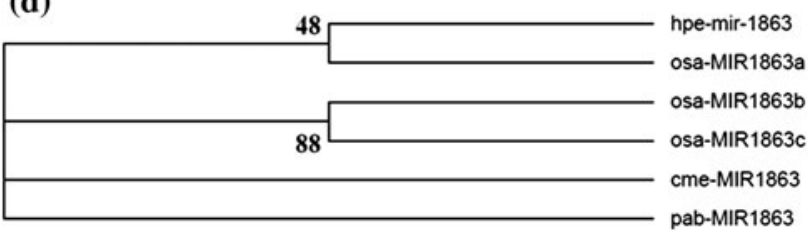

Fig. 5 Phylogenetic analysis of four large families of precursor miRNAs a phylogenetic tree for mir-156, $\mathbf{b}$ phylogenetic tree for mir169 , c phylogenetic tree for mir-395, d phylogenetic tree for mir-1863

to defense mechanism, conferring tolerance to stress response and signal transduction. Role of these novel miRNAs in regulation of plant metabolism could be determined by metabolic modeling (Gupta et al. 2012a, b). Further research work with identified miRNAs can provide insight into the role of post-transcriptional gene silencing in the biotic and abiotic stress responses.

H. petiolaris is a less studied plant member of Asteraceae family, having vast medicinal value. This effort will accelerate the research in $H$. petiolaris structural and functional genomics. Genomic features and significant information can be mined using different algorithms and statistical approaches (Nounou et al. 2013, Singh et al. 2013). As genome data and bioinformatics resources increase rapidly in size and complexity, there is need to develop the methods for analysis and extraction of useful information from data as well as for understanding of biological processes. There exists great possibility in mining the huge information from databases and transfer that to divergent plant species.

\section{Conclusion}

Eleven potential miRNAs and their targets were identified in prairie sunflower, one of the most important crops around the world. Most of the identified miRNA targets are involved in stress responses like alpha-trehalose phosphate synthase that controls the over expression of citric acid, ATP sulfurylase participate to enhance the self defense mechanism. The findings suggest that sunflower miRNA is involved in the regulation of genes related to defense mechanism, conferring tolerance to stress response and signal transduction. Further research work with identified miRNAs can provide insight into the role of post-transcriptional gene silencing in the biotic and abiotic stress responses. Results further suggest that valuable information can be mined from genome and EST sequences freely available in public databases.

\section{References}

Ambros V, Bartel B, Bartel DP, Burge CB, Carrington JC, Chen X, Dreyfuss G, Eddy SR, Griffiths-Jones S, Marshall M, Matzke M, Ruvkun G, Tuschl T (2003) A uniform system for microRNA annotation. RNA 9:277-279

Bartel DP, Chen CZ (2004) Micromanagers of gene expression: the potentially widespread influence of metazoan microRNAs. Nat Rev Genet 5:396-400

Boguski MS, Lowe TM, Tolstoshev CM (1993) dbEST-database for expressed sequence tags. Nat Genet 4:332-333

Etheridge A, Lee I, Hood L, Galas D, Wang K (2011) Extracellular microRNA: a new source of biomarkers. Mutat Res 717:85-90

Griffiths-Jones S, Grocock RJ, van Dongen S, Bateman A, Enright AJ (2006) miRBase: microRNA sequences, targets and gene nomenclature. Nucleic Acids Res 34:D140-D144

Gupta MK, Agarwal K, Prakash N, Singh DB, Misra K (2012a) Prediction of miRNA in HIV-1 genome and its targets through artificial neural network: a bioinformatics approach. Netw Model Anal Health Inf Bioinform 1:141-151

Gupta MK, Singh DB, Rath SK, Misra K (2012b) Metabolic modeling and simulation analysis of thyroid disorder pathway. J Comput Sci Syst Biol 5:52-61

Hall TA (1999) BioEdit: a user-friendly biological sequence alignment editor and analysis program for Windows 95/98/NT. Nucl Acids Symp Ser 41:95-98

Jha A, Shankar R (2011) Employing machine learning for reliable miRNA target identification in plants. BMC Genomics 12:636 
Kong Y, Han JH (2005) MicroRNA: biological and computational perspective. Genomics Proteomics Bioinformatics 3:62-72

Kozomara A, Griffiths-Jones S (2011) miRBase: integrating microRNA annotation and deep-sequencing data. Nucleic Acids Res 39:D152-D157

Krzyzanowski PM, Price FD, Muro EM, Rudnicki MA, AndradeNavarro MA (2011) Integration of expressed sequence tag data flanking predicted RNA secondary structures facilitates novel non-coding RNA discovery. PLoS One 6:e20561

Lawrence CL, Botting CH, Antrobus R, Coote PJ (2004) Evidence of a new role for the high-osmolarity glycerol mitogen-activated protein kinase pathway in yeast: regulating adaptation to citric acid stress. Mol Cell Biol 24:3307-3323

Lee RC, Feinbaum RL, Ambros V (1993) The C. elegans heterochronic gene lin-4 encodes small RNAs with antisense complementarity to lin-14. Cell 75:843-854

Lee Y, Ahn C, Han J, Choi H, Kim J, Yim J, Lee J, Provost P, Rådmark O, Kim S, Kim VN (2003) The nuclear RNase III Drosha initiates microRNA processing. Nature 425:415-419

Liang C, Zhang X, Zou J, Xu D, Su F, Ye N (2010) Identification of miRNA from Porphyra yezoensis by high-throughput sequencing and bioinformatics analysis. PLoS One 5:e10698

Lu Y, Yang X (2010) Computational identification of novel microRNAs and their targets in Vigna unguiculata. Comp Funct Genomics. doi:10.1155/2010/128297 (pii: 128297)

Masoudi-Nejad A, Tonomura K, Kawashima S, Moriya Y, Suzuki M, Itoh M, Kanehisa M, Endo T, Goto S (2006) EGassembler: online bioinformatics service for large-scale processing, clustering and assembling ESTs and genomic DNA fragments. Nucleic Acids Res 34:W459-W462

Miller JF, Gulya TJ (2001) Registration of three rust resistant sunflower germplasm populations. Crop Sci 41:601

Nounou MN, Nounou HN, Mansouri M (2013) Model-based and model-free filtering of genomic data. Netw Model Anal Health Inform Bioinform 2:109-121

Pfeffer S, Voinnet O (2006) Viruses, microRNAs and cancer. Oncogene 25:6211-6219

Philip HW, Rod E, Georg W (2012) Plant microRNA prediction by supervised machine learning using c5.0 decision trees. J Nucleic Acids. doi:10.1155/2012/652979

Rausch T, Wachter A (2005) Sulfur metabolism: a versatile platform for launching defence operations. Trend Plant Sci 10:503-509

Sahu S, Dixit R (2011) In silico miRNAs target identification in Burma agrimony. Int J Contemp Res Eng Technol 1:82

Sahu S, Khushwaha A, Dixit R (2011) Computational identification of miRNA in medicinal plant Senecio vulgaris (Groundsel). Bioinformation 7:375-378
Seiler GJ (2010) Utilization of wild Helianthus species in breeding for disease resistance. In: Proceedings of the International Sunflower Association (ISA) symposium sunflower breeding on resistance to diseases, pp 36-50

Shallu K, Sahu S (2011) In silico miRNA identification and target prediction in HPV. Indian J Sci Res 2:44

Singh DB, Gupta MK, Kesharwani RK, Misra K (2013) Comparative docking and ADMET study of some curcumin derivatives and herbal congeners targeting $\beta$-amyloid. Netw Model Anal Health Inform Bioinform 2:13-27

Suárez R, Wong A, Ramírez M, Barraza A, Orozco Mdel C, Cevallos MA, Lara M, Hernández G, Iturriaga G (2008) Improvement of drought tolerance and grain yield in common bean by overexpressing trehalose-6-phosphate synthase in rhizobia. Mol Plant Microbe Interact 21:958-966

Tamura K, Peterson D, Peterson N, Stecher G, Nei M, Kumar S (2011) MEGA5: molecular evolutionary genetics analysis using maximum likelihood, evolutionary distance, and maximum parsimony methods. Mol Biol Evol 28:2731-2739

Varkonyi-Gasic E, Gould N, Sandanayaka M, Sutherland P, MacDiarmid RM (2010) Characterisation of microRNAs from apple (Malus domestica 'Royal Gala') vascular tissue and phloem sap. BMC Plant Biol 10:159

Wegener D, Rossi S, Buffa F, Delorenzi M, Rüping S (2013) Towards an environment for data mining based analysis processes in bioinformatics and personalized medicine. Netw Model Anal Health Inform Bioinform 2:29-44

Xie FL, Huang SQ, Guo K, Xiang AL, Zhu YY, Nie L, Yang ZM (2007) Computational identification of novel microRNAs and targets in Brassica napus. FEBS Lett 581:1464-1474

Xie F, Frazier TP, Zhang B (2010) Identification and characterization of microRNAs and their targets in the bioenergy plant switchgrass (Panicum virgatum). Planta 232:417-434

Yao Y, Guo G, Ni Z, Sunkar R, Du J, Zhu JK, Sun Q (2007) Cloning and characterization of microRNAs from wheat (Triticum aestivum L.). Genome Biol 8:R96

Yeo JH, Chong MM (2011) Many routes to a micro RNA. IUBMB Life 63:972-978

Zhang BH, Pan XP, Cox SB, Cobb GP, Anderson TA (2006) Evidence that miRNAs are different from other RNAs. Cell Mol Life Sci 63:246-254

Zhao T, Li G, Mi S, Li S, Hannon GJ, Wang XJ, Qi Y (2007) A complex system of small RNAs in the unicellular green alga Chlamydomonas reinhardtii. Genes Dev 21:1190-1203

Zuker M (2003) Mfold web server for nucleic acid folding and hybridization prediction. Nucleic Acids Res 31:3406-3415 